Structural dynamics in phospholipid bilayers from deuterium spin-lattice relaxation time measurements

Michael F. Brown and Joachim SeeligUlrich Häberlen

Citation: The Journal of Chemical Physics 70, 5045 (1979); doi: 10.1063/1.437346

View online: $\mathrm{http}: / / \mathrm{dx}$.doi.org/10.1063/1.437346

View Table of Contents: http://aip.scitation.org/toc/jcp/70/11

Published by the American Institute of Physics

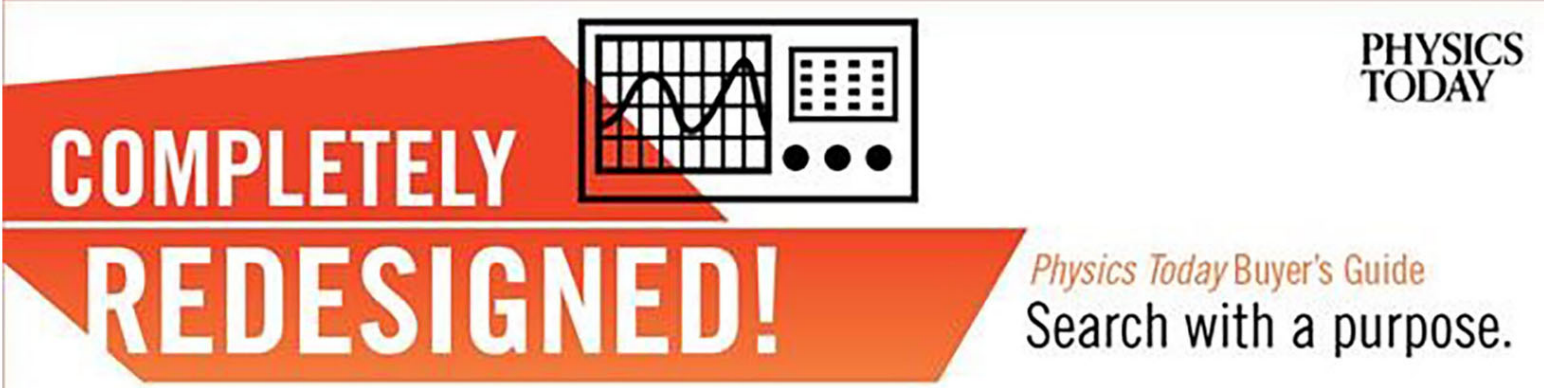




\title{
Structural dynamics in phospholipid bilayers from deuterium spin-lattice relaxation time measurements ${ }^{a)}$
}

\author{
Michael F. Brown ${ }^{\text {b) }}$ and Joachim Seelig ${ }^{c}$ \\ Abt. Biophysikalische Chemie, Biozentrum der Universität Basel, Klingelbergstrasse 70, CH-4056 \\ Basel, Switzerland \\ Ulrich Häberlen \\ Abt. Molekulare Physik, Max-Planck-Institut für Medizinische Forschung, Jahnstrasse 29, D-6900 \\ Heidelberg, West Germany \\ (Received 22 November 1978)
}

\begin{abstract}
The quadrupolar spin-lattice $\left(T_{1}\right)$ relaxation of deuterium labeled phospholipid bilayers has been investigated at a resonance frequency of $54.4 \mathrm{MHz}$. $T_{1}$ measurements are reported for multilamellar dispersions, single bilayer vesicles, and chloroform/methanol solutions of 1,2-dipalmitoyl-sn-glycero-3phosphocholine (DPPC), selectively deuterated at ten different positions in each of the fatty acyl chains and at the $s n-3$ carbon of the glycerol backbone. At all segment positions investigated, the $T_{1}$ relaxation times of the multilamellar and vesicle samples of DPPC were found to be similar. The profiles of the spin-lattice relaxation rate $\left(1 / T_{1}\right)$ as a function of the deuterated chain segment position resemble the previously determined order profiles [A. Seelig and J. Seelig, Biochem. 13, 4839 (1974)]. In particular, the relaxation rates are approximately constant over the first part of the fatty acyl chains (carbon segments $\mathrm{C} 3-\mathrm{C} 9$ ), then decreasing in the central region of the bilayer. In chloroform/methanol solution, by contrast, the relaxation rates decrease continuously from the glycerol backbone region to the chain terminal methyl groups. The contributions from molecular order and motion to the $T_{1}$ relaxation rates have been evaluated and correlation time profiles derived as a function of chain position. The results suggest that the motions of the various methylene segments are correlated in the first part of the fatty acyl chains (C3-C9), occurring at frequencies up to $1 / \tau_{c} \simeq 10^{10} \mathrm{~Hz}$. Beyond $\mathrm{C} 9$, the rate and amplitude of the chain segmental motions increase, approaching that of simple paraffinic liquids in the central region of the bilayer $\left(1 / \tau_{c} \simeq 10^{11} \mathrm{~Hz}\right)$. The $T_{1}$ relaxation rates of multilamellar dispersions of 1,2-dioleoyl-sn-glycero3-phosphocholine (DOPC) deuterated at the 9, 10 double bond of the $s n-2$ chain were also determined and found to be significantly faster than those of the $\mathrm{CD}_{2}$ chain segments of DPPC bilayers. This is most likely due to the larger size and correspondingly slower motion of the chain segment containing the double bond. At segments close to the lipid-water interface the rate of motion is considerably less than in the hydrocarbon region of the bilayer.
\end{abstract}

\section{INTRODUCTION}

An understanding of the conformation and dynamic state of lipid bilayers is of interest from both a physical and a biological perspective. Two experimental systems have been studied extensively: multilamellar dispersions of phospholipids in water ${ }^{1}$ and suspensions of small, single bilayer vesicles formed by sonication. ${ }^{2}$ From a phys ical point of view, lipid bilayers are interesting in that they share a number of features in common with liquid crystals and have been the focus of recent efforts to explain the ordering and phase transitions of smectic mesophases..$^{3-8}$ In biological systems, lipids are found primarily as constituents of cell membrances. Consequently, studies of model bilayers and their interaction with anesthetics, ${ }^{9-11}$ ions, ${ }^{12-15}$ cholesterol, ${ }^{16-18}$ and proteins ${ }^{19-24}$ may be helpful in elucidating certain aspects of cellular function.

In analyzing the physical properties of bilayer membrances one must differentiate between (i) the ordering

\footnotetext{
${ }^{\text {a }}$ Work supported by Grant 3.008.76 from the Swiss National Science Foundation and by the Sandoz Foundation for the Promotion of Biomedical Sciences.

b) Postdoctoral fellow of the U. S. National Institute of General Medical Sciences. Present address: Department of Chemistry, University of California, Berkeley, CA 94720.

c) To whom correspondence should be addressed.
}

of the phospholipid molecules, i. e., the time averaged orientation and amplitude of the angular excursions of particular groups within the hydrocarbon chains and the head group region and (ii) the rate of motion of the various segments. Both of these quantities are accessible to study us ing NMR methods. The degree of motional averaging of various static interactions provides information related to the average molecular conformation, while analysis of the nuclear relaxation rates will establish the time scale of the molecular motions. A method which has proven fruitful in these respects is deuterium NMR. ${ }^{25,26}$ The residual quadrupole splittings of selectively deuterated lipid bilayers can be simply related to the order parameters of the various flexible molecular segments and have been the subject of a number of recent studies. ${ }^{27-39}$ The dynamic information obtainable from deuterium relaxation time measurements, however, has not yet been systematically investigated in studies of lipid bilayers.

The purpose of this paper is to present some recently obtained spin-lattice $\left(T_{1}\right)$ relaxation time data for phospholipid bilayers that have been selectively deuterated at a number of different segment positions and to discuss the results in terms of molecular motion. The problem of the fatty acyl chain motion in phospholipid bilayers has been investigated by a number of workers. ${ }^{34-38}$ However, progress in this area has been ham- 
pered by at least two factors: (i) the lack of well-defined experimental data regarding the dependence of $T_{1}$ on the chain segment position and (ii) the lack of an appropriate theoretical analysis of the $T_{1}$ data in terms of the chain ordering and rate of motion. The most complete $T_{1}$ data obtained to date are from carbon-13 NMR studies, ${ }^{35,38}$ but even in this case the information is not sufficient to unambiguously define the nature of the chain mobility. This is partly because individual resonances can be resolved in natural abundance carbon-13 NMR spectra only from those carbons near the beginning or end of the fatty acyl chains, so that isotopic labeling procedures are necessary. This has been attempted in only one instance.$^{39}$ As a result, the interpretation of the presently available NMR relaxation data has remained problematic and has led to a number of rather complex models. ${ }^{40-43}$

We have adopted a somewhat different approach and have analyzed the more extensive deuterium $T_{1}$ data in terms of a simple stochastic model for the motion of individual chain segments in lipid bilayers. Using our approach, we are able to quantitatively assess the dependence of the relaxation rates on order and thereby determine rotational correlation time profiles as a function of chain segment position. We find that the rate of motion, rather than the ordering, is the dominant factor determining the relaxation of the systems studied here. In this article, we discuss the motional profile in the hydrocarbon region of phospholipid bilayers and compare it to the previously determined profiles of the deuterium order parameter as a function of chain position. We are also able to provide information regarding the behavior of segments close to the aqueous interface of lipid bilayers, and on the motional state of lipids in organic solution compared to unsonicated multilamellar dispersions and sonicated vesicles.

\section{ANALYSIS OF DEUTERIUM RELAXATION IN LIPID BILAYERS}

The relaxation pathway of deuterium is primarily determined by the quadrupolar interaction, which is generally much stronger than the dipolar coupling involving adjacent nuclei. The quadrupolar relaxation mechanism is intramolecular and due to rotational reorientation of the molecule-fixed electric field gradient tensor with respect to the applied magnetic field. For aliphatic carbon-deuterium bonds, which are considered here, the field gradient is approximately axially symmetric about the bond axis. ${ }^{26}$

In ordered systems such as lipid bilayers, molecular motions are restricted and the quadrupolar interaction is not averaged over all space. In such cases, the interaction Hamiltonian is most usefully separated into its time averaged and fluctuating components

$$
\mathfrak{H}_{Q}(t)=\overline{\mathfrak{H}_{Q}}+\mathcal{H}_{Q}^{\prime}(t) \text {. }
$$

The time averaged Hamiltonian $\overline{K_{0}}$ is related to the residual deuterium quadrupole splitting, while the fluctuating part $\mathcal{K C}_{0}^{\prime}(t)$ produces relaxation. In isotropic or anisotropic solutions $\overline{\mathfrak{F}_{0}}$ is zero (no quadrupole splitting observable) and consequently the entire quadrupolar Hamiltonian contributes to the relaxation. In ordered systems
$\overline{\mathcal{F}_{Q}}$ retains some finite value and the fluctuating part is correspondingly reduced.

In general, the relaxation in lipid bilayers will depend on at least three parameters: (i) the correlation time(s) for the molecular motion, (ii) the order parameter(s) characterizing the extent of restriction of the molecular fluctuations, and (iii) the orientation of the normal to the lipid bilayer surface (known as the director, an axis of rotational symmetry) with respect to the applied magnetic field. In order to quantitatively analyze the deuterium spin-lattice $\left(T_{1}\right)$ relaxation times, we have used the following approach. The spin-lattice relaxation rate, $1 / T_{1}$, of a spin one system such as deuterium is given by $^{44}$

$$
\frac{1}{T_{1}}=W_{1}+2 W_{2}
$$

where $W_{1}$ and $W_{2}$ are the transition probabilities for the $|0\rangle-| \pm 1\rangle$ and $|-1\rangle-|+1\rangle$ spin transitions. Strictly speaking, Eq. (2) is correct only for degenerate transitions and we have therefore neglected any small shifts of the magnetic energy levels due to the residual quadrupolar interaction. The transition probabilities depend on the strength of the fluctuating quadrupolar interaction near the resonance frequency $\omega_{0}$ and at $2 \omega_{0}$. For the present, we have employed a relatively simple motional model, in which the carbon-deuterium bond vector is assumed to undergo statisticaliy random fluctuations of limited amplitude with respect to the bilayer normal. We assume (i) that the autocorrelation function for the carbon-deuterium bond fluctuations giving $r$ ise to the $T_{1}$ relaxation decays with a single exponential time constant $\tau_{c}$, i. e., we neglect any anisotropy in the rate of motion of individual chain segments and (ii) that these motions fall into the extreme narrowing limit $\left(\omega_{0}^{2} \tau_{c}^{2} \ll 1\right)$. With these assumptions the transition probabilities $W_{1 m}$ are given by ${ }^{44}$

$$
\begin{aligned}
W_{l m} & =\frac{2}{\hbar^{2}} \overline{\left|\left\langle l\left|\mathcal{K}_{Q}^{\prime}(t)\right| m\right\rangle\right|^{2}} \tau_{c} \\
& =\frac{2}{\hbar^{2}}\left\{\overline{\left|\left\langle l\left|\mathfrak{K}_{Q}(t)-\overline{\mathfrak{H}_{Q}}\right| m\right\rangle\right|^{2}}\right\} \tau_{c} \\
& =\frac{2}{\hbar^{2}}\left\{\overline{\left.|l| \mathfrak{H}_{Q}(t)|m\rangle\right|^{2}}-\left|\left\langle l\left|\overline{\mathfrak{H}_{Q}}\right| m\right\rangle\right|^{2}\right\} \tau_{c} .
\end{aligned}
$$

It is through $\mathcal{K}_{Q}^{\prime}(t)$ that the ordering of the system enters into the relaxation time expressions. For lipid bilayers $\overline{\mathcal{F}_{Q}}$ depends on both the director orientation and the order parameter of the carbon-deuterium bond, defined by

$$
S_{\mathrm{CD}}=\frac{1}{2}\left(3 \overline{\cos ^{2} \beta(t)}-1\right),
$$

where $\beta(t)$ is the time dependent angle between the carbon-deuterium bond vector and the bilayer normal. In evaluating Eqs. (3) we have made use of standard procedures for the analysis of relaxation in liquid crystals, ${ }^{45}$ that is, representation of the quadrupolar Hamiltonian $\mathcal{K}_{Q}^{\prime}(t)$ using irreducible tensors and performing the appropriate coordinate transformations by means of Wigner rotation matrices. ${ }^{46}$ After some lengthy algebra, the following expression for the spin-lattice relaxation time is obtained 


$$
\frac{1}{T_{1}}=\frac{3}{8}\left(\frac{e^{2} q Q}{\hbar}\right)^{2}\left[1-S_{\mathrm{CD}} S_{\beta^{\prime}}-S_{\mathrm{CD}}^{2}\left(1-S_{\beta^{\circ}}\right)\right] \tau_{c} .
$$

In the above equation $\left(e^{2} q Q / h\right)$ denotes the static quadrupole coupling constant $(170 \mathrm{kHz}$ for carbon-deuterium bonds ${ }^{26}$ ) and $S_{\beta}$, is a macroscopic orientation factor given by

$$
S_{\beta^{\prime}}=\frac{1}{2}\left(3 \cos ^{2} \beta^{\prime}-1\right),
$$

where $\beta^{\prime}$ is the angle between the bilayer normal and the magnetic field.

The deuterium NMR spectra observed from unoriented multilamellar dispersions correspond to a random distribution of director orientations and consequently Eq. (5) predicts that $T_{1}$ will vary across the spectrum according to the factor $S_{\beta^{\prime}}$. How ever, the predominant features of the powder-type spectra are the sharp edges corresponding to domains where the director is oriented perpendicularly to the magnetic field $\left(\beta^{\prime}=90^{\circ}, S_{\beta^{\prime}}=-\frac{1}{2}\right)$. Thus, in terms of our motional model, the spin-lattice relaxation rate for multilamellar dispersions is given by

$$
\left(\frac{1}{T_{1}}\right)_{B^{\prime}=90}=\frac{3}{8}\left(\frac{e^{2} q Q}{\hbar}\right)^{2}\left(1+\frac{1}{2} S_{\mathrm{CD}}-\frac{3}{2} S_{\mathrm{CD}}^{2}\right) \tau_{c} .
$$

For the case of small, single-bilayer vesicles, the time average of the quadrupolar Hamiltonian over the rapid internal motions, namely $\overline{\mathcal{K}_{Q}}$, is further modulated by the relatively slow vesicle tumbling $\left(\tau_{v} \cong 10^{-6} \mathrm{sec}\right)$, leading to motional averaging of the deuterium powder pattern to a single Lorentzian spectral line. ${ }^{29}$ Since the rate of vesicle tumbling is still much faster than the $T_{1}$ relaxation times, which are in the msec time range, $S_{\beta^{\prime}}$ is averaged to zero and the relaxation rate is given $b y^{26}$

$$
\left(\frac{1}{T_{1}}\right)_{\text {vesicles }}=\frac{3}{8}\left(\frac{e^{2} q Q}{\hbar}\right)^{2}\left(1-S_{\mathrm{CD}}^{2}\right) \tau_{c} .
$$

The above formula [Eq. (8)] has been used previously for bilayers of potassium palmitate by Davis and coworkers. ${ }^{47}$ A more comprehensive account of deuterium relaxation in lipid bilayers based on the density matrix formalism is given elsewhere. ${ }^{48}$

\section{EXPERIMENTAL}

Selectively deuterated 1,2-dipalmitoyl-sn-glycero-3phosphocholine (DPPC) ${ }^{49}$ and 1,2-dioeloyl -sn-glycero3-phosphocholine (DOPC) were a gift of A. Seelig. ${ }^{28,30}$ The multilamellar samples were prepared by dispersal of approximately $20-100 \mathrm{mg}$ of phospholipid in water containing $10^{-4} \mathrm{M}$ EDTA at a concentration of $50 \mathrm{wt} . \%$. Since the relaxation rates investigated are short and no differences were noted between samples that were argon purged and those containing atmospheric oxygen, we did not routinely deoxygenate the samples. Single walled vesicles were prepared by sonication of approximately $10-20 \mathrm{mg}$ of phospholipid in $0.5 \mathrm{ml}$ of water containing $10^{-4}$ EDTA, under a nitrogen atmosphere, using a Branson B-12 sonifier equipped with a microtip. The samples were maintained in a water bath at $50^{\circ} \mathrm{C}$ during sonication and were never allowed to cool below the gel to liquid crystalline phase transition temperature $\left(41^{\circ} \mathrm{C}\right)$ for the duration of the experiments. After use the sam- ples gave single spots upon thin layer chromatography in chloroform/methanol/water $(65: 35: 4)$.

The NMR studies employed an 8.32 T Oxford Instruments cryomagnet (deutérium frequency $54.4 \mathrm{MHz}$ ), with standard Bruker pulse hardware interfaced to an in-house computer system. The $T_{1}$ relaxation times of DPPC dissolved in 9:1 chloroform/methanol were measured by the standard inversion recovery pulse method $\left(180^{\circ}-\tau-90^{\circ}\right)$. In all cases complete signal inversion was observed. The $T_{1}$ relaxation times of the multilamellar and vesicle samples were measured by the progressive saturation method $\left(90^{\circ}-\tau-90^{\circ}\right)$, using the Fourier transform of either the normal free induction decay or the second half of the quadrupolar echo ${ }^{50}$ to establish the decay rates. No homospoil pulses were used between successive $90^{\circ}$ pulses, since for these systems $T_{2} \ll T_{1}$. In most instances it was possible to observe complete resonance saturation within the signal to noise level of the spectra. The pulse angles were carefully adjusted using the free induction decay of $\mathrm{D}_{2} \mathrm{O}$. A $90^{\circ}$ pulse was generally found to be about $4 \mu \mathrm{sec}$ in duration.

The major sources of systematic error in determining $T_{1}$ using the progressive saturation method are (i) errors in the pulse (flip) angle and (ii) errors in determination of the infinity magnetizations used to analyze the decay rates. The latter problem can be minimized by curve fitting the exponential recovery curves rather than the first order decay plots; however, for the present case we have generally determined $4-5$ infinity values and used their average in determining $T_{1}$ from plots of $\ln \left(M_{0}-M_{g}\right)$ vs $\tau$ in the customary manner. A weighting factor of $\left(M_{0}-M_{\varepsilon}\right)^{2}$ was used in the least squares fitting procedure to properly account for the nonlinear instrumental error. We have minimized pulse angle errors by adjusting the pulse frequency to the center of the quadrupole doublet, so that the spectra are folded back and the maximum half-quadrupole splittings are less than $15 \mathrm{kHz}$. This leads to maximum systematic pulse errors of up to $20^{\circ}$, corresponding to a systematic error in the $T_{1}$ values of $12 \% .^{51}$ For half-quadrupole splittings smaller than $15 \mathrm{kHz}$ the $T_{1}$ values are correspondingly more accurate. Since the contributions from systematic errors are small, we have only considered random errors in analyzing the $T_{1}$ data. The quoted error limits refer to \pm the standard deviation of the mean.

\section{RESULTS}

Some typical semilogarithmic magnetization recovery plots obtained for multilamellar dispersions of DPPC specifically deuterated in each of the two hydrocarbon chains are shown in Fig. 1. In all cases investigated, the quadrupole splittings of the various DPPC bilayer phases were in agreement with previous results ${ }^{28,52,53}$ and the relaxation was exponential. At those segment positions where the $s n-1$ and $s n-2$ chains give rise to dif ferent quadrupole splittings, ${ }^{28}$ such as the $\mathrm{C} 2$ position, ${ }^{16,52}$ similar spin-lattice relaxation times were observed. Figure 2 shows bilayer profiles of the relaxation rate, $1 / T_{1}$, as a function of the deuterated segment position. The relaxation rates appear to be more or less constant over the first half of the fatty acyl chains 


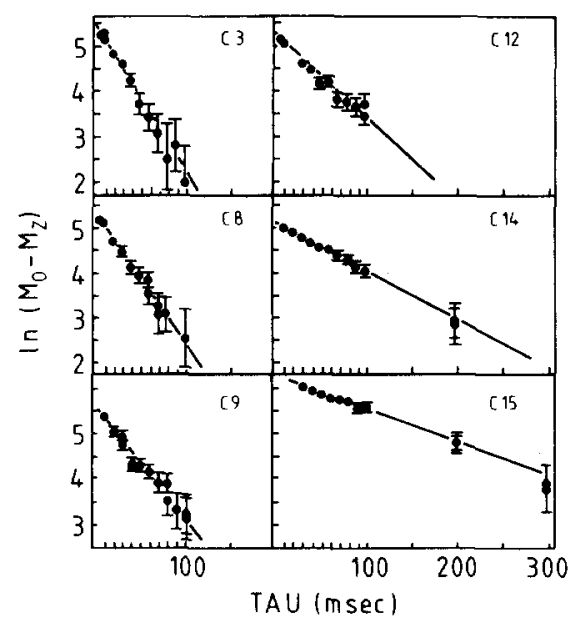

FIG. 1. Measurement of the spin-lattice $\left(T_{1}\right)$ relaxation times of selectively deuterated DPPC multilamellar dispersions using the saturation recovery method at $51{ }^{\circ} \mathrm{C}$. The data are expressed as plots of $\ln \left(M_{0}-M_{z}\right)$ vs $\tau$, where $M_{0}$ is the equilibrium magnetization at long pulse intervals and $M_{z}$ is the steady state magnetization at a shorter pulse interval $\tau$. The position of the deuterium labels in the fatty acyl chains is indicated by $\mathrm{C} n$.

(C3 to about C9), followed by a decrease in the central region of the bilayer (C10-C15). These results agree fairly well with a recent study of Davis ${ }^{54}$ using perdeuterated DPPC. Distinctly faster relaxation rates are observed for the glycerol backbone $s n-3$ segment (G3 in our nomenclature) and for the fatty acyl chain segments immediately adjacent to the glycerol groups (C2).

The deuterium $T_{1}$ relaxation rates of the DPPC vesicle solutions were generally observed to be exponential, except at the C2 position, where the slightly nonexponential relaxation may be related to the fact that three components are observed in the deuterium NMR spectra of the corresponding unsonicated dispersions. ${ }^{16,52}$ A com-

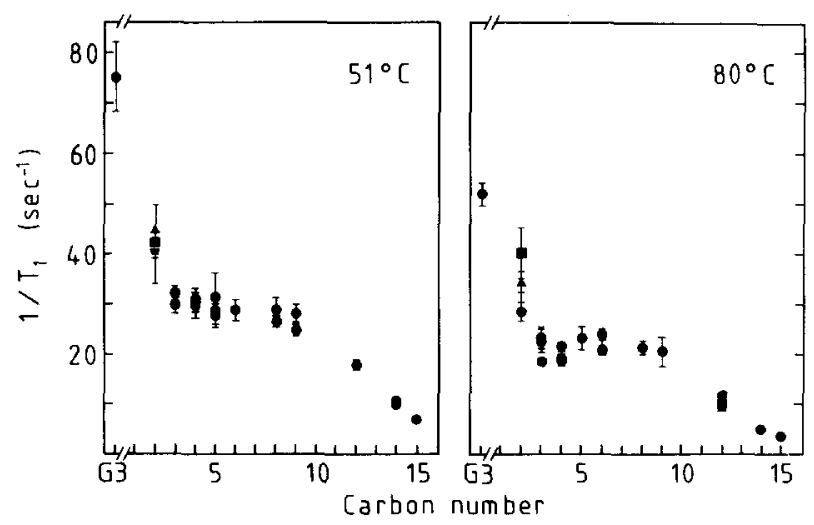

FIG. 2. Plots of the spin-lattice relaxation rates $\left(1 / T_{1}\right)$ of DP PC multilamellar dispersions as a function of the deuterated segment position at 51 and $80^{\circ} \mathrm{C}$. At the $\mathrm{C} 2$ position three resonances are observed ${ }^{52}$; the triangles, squares, and circles refer to the $s n-2 a$ (smaller quadrupole splitting), $s n-2 b$ (larger quadrupole splitting), and $s n-1$ chain resonances, respectively. The multiple data points at the other segment positions indicate multiple $T_{1}$ measurements. Data are also shown for DPPC deuterated at the $s n-3$ carbon of the glycerol moiety, indicated by G3.

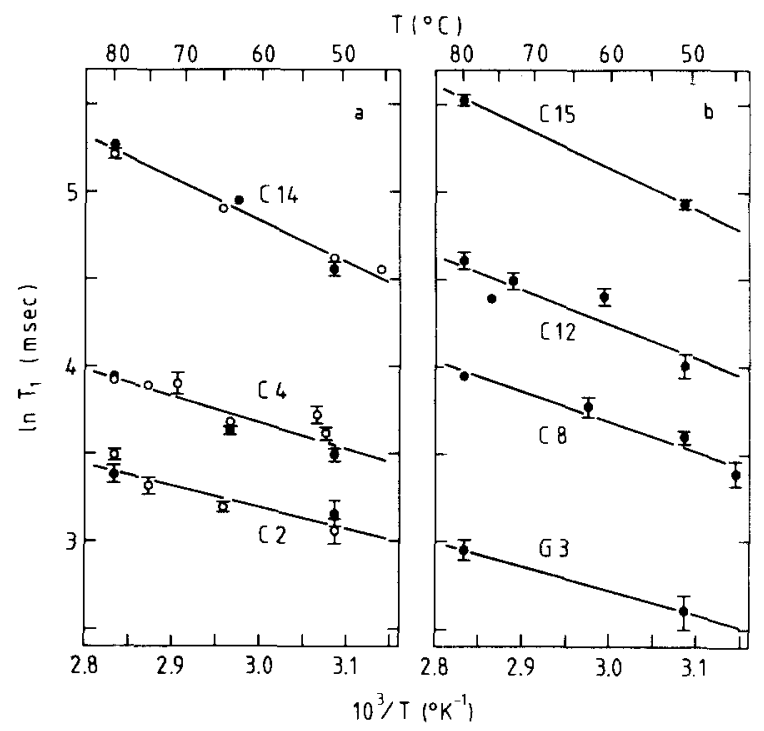

FIG. 3. Arrhenius plots of the $T_{1}$ relaxation times. Figure 3(a) shows a comparison of the data obtained for DPPC multilamellar dispersions $(\bullet)$ and sonicated vesicles $(0)$, deuterated at the indicated segment positions. The data for the $\mathrm{C} 2$ position of the multilamellar dispersions refer to the average of the three observed resonances. ${ }^{52}$ Figure $3(\mathrm{~b})$ shows additional data for the DPPC multilamellae.

parison of the relaxation data obtained for sonicated vesicles and multilamellar dispersions of DPPC is shown in Fig. 3(a), where the data are expressed as Arrhenius plots of $\ln T_{1}$ vs reciprocal temperature. Statistically significant differences in the $T_{1}$ relaxation times of the vesicle and multilamellar preparations could not be detected within the accuracy of our experiments. Figure 3 (b) shows some additional temperation dependences for the multilamellar DPPC samples at several other segment positions. The least squares slopes of the Arrhenius plots shown in Fig. 3 correspond to an average activation energy of $14.6 \pm 1.3 \mathrm{~kJ} \mathrm{~mol}^{-1}(3.5 \pm 0.3 \mathrm{kcal}$ $\left.\mathrm{mol}^{-1}\right)$, in good agreement with previous proton and carbon-13 NMR studies. $34,36,39$ Within the error of our measurements, we were not able to discern any dependence of the activation energies on the position of the deuterated chain segment. Such a dependence has been previously reported for the lamellar phase of potassium palmitate. ${ }^{47}$

Figure 4 depicts the results of $T_{1}$ relaxation studies of specifically deuterated DPPC dissolved in chloroform/ methanol $(9: 1 \mathrm{vol} / \mathrm{vol})$. At all segment positions investigated the relaxation was observed to be exponential. The relaxation rates in chloroform/methanol decrease continuously along the fatty acyl chains and are significantly smaller than those of the corresponding multilamellar and vesicle dispersions of DPPC. Figure 5 shows Arrhenius plots of the $T_{1}$ data in $9: 1$ chloroform/ methanol solution. The activation energy in this solvent is $10.5 \pm 2.1 \mathrm{~kJ} \mathrm{~mol}^{-1}\left(2.5 \pm 0.5 \mathrm{kcal} \mathrm{mol}^{-1}\right)$. No dependence of the activation energies on the chain segment position was noted.

The $T_{1}$ data for the DPPC multilamellar dispersions, vesicles, and in $9: 1$ chloroform/methanol solution are 


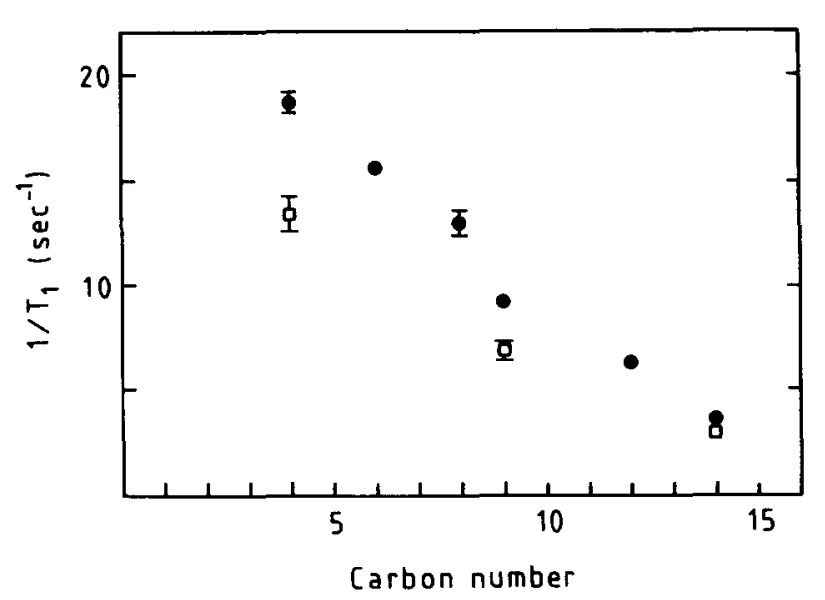

FIG. 4. Spin-lattice relaxation of DPPC in $9: 1$ chloroform/ methanol as a function of the deuterated segment position at $23^{\circ} \mathrm{C}(\bullet)$ and $37^{\circ} \mathrm{C}(0)$. The inversion recovery method was used to determine the $T_{1}$ relaxation times.

summarized in Table I. Some preliminary data for multilamellar samples of 1,2-dioleoyl-sn-glycero-3-phosphocholine (DOPC) are also included. Although the two deuterons of the double bond give rise to quite different quadrupole splittings, ${ }^{32}$ differences in the $T_{1}$ relaxation rates could not be detected (activation energy $=14.2$ $\pm 1.7 \mathrm{~kJ} \mathrm{~mol}^{-1}$ ). For all the systems investigated, the $T_{1}$ relaxation times increase with temperature, suggesting that the relevant molecular motions are in the short correlation time regime $\left(\omega_{0}^{2} \tau_{c}^{2} \ll 1\right)$.

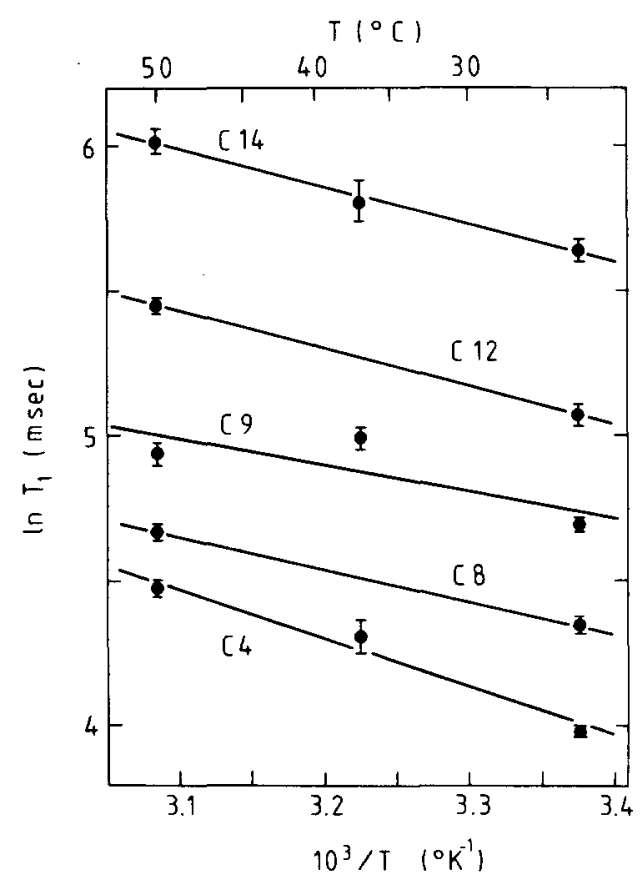

FIG. 5. Arrhenius plots of the $T_{1}$ relaxation times of DPPC in $9: 1$ chloroform/methanol, deuterated as indicated.

\section{DISCUSSION}

\section{A. Dependence of the $T_{1}$ relaxation times on the segmental order parameter $S_{C D}$}

Using deuterium magnetic resonance, two independent parameters can be derived which provide insight into the

TABLE I. Spin-lattice relaxation times of selectively deuterated DPPC and DOPC. ${ }^{a} T_{1}(\mathrm{msec}) \pm \mathrm{std}$. dev.

\begin{tabular}{|c|c|c|c|c|c|c|}
\hline \multirow{2}{*}{$\begin{array}{l}\begin{array}{l}\text { Segment } \\
\text { position }^{\mathrm{b}}\end{array} \\
\text { DPPC }\end{array}$} & \multicolumn{2}{|c|}{ Multibilayers } & \multicolumn{2}{|c|}{ Vesicles } & \multicolumn{2}{|c|}{$9: 1 \mathrm{CHCl}_{3}: \mathrm{MeOH}$} \\
\hline & $51^{\circ}$ & $80^{\circ}$ & $51^{\circ}$ & $80^{\circ}$ & $23^{\circ}$ & $51^{\circ}$ \\
\hline G3 & $13.3 \pm 1.4$ & $19.1 \pm 1.2$ & $\cdots$ & $\cdots$ & $\ldots$ & $\ldots$ \\
\hline $\mathrm{C} 2$ & $23.4+3.3^{c}$ & $29.6 \pm 2.6^{c}$ & $21.2+1.7^{\mathrm{d}}$ & $32.7 \pm 1.1^{d}$ & $\ldots$ & $\ldots$ \\
\hline C3 & $32,2 \pm 1.4$ & $46.9 \pm 1.7$ & $\cdots$ & $\infty$ & $\cdots$ & $89.2 \pm 3.5$ \\
\hline $\mathrm{C} 4$ & $32.7 \pm 1.2$ & $50.8 \pm 1.1$ & $39.2 \pm 1.2$ & $51.1 \pm 1.0$ & $53.6 \pm 1.1$ & $87.7 \pm 1.5$ \\
\hline C5 & $33.0 \pm 2.1$ & $43.1 \pm 4.7$ & $\cdots$ & $\cdots$ & $\cdots$ & $\cdots$ \\
\hline C6 & $34.3 \pm 1.8$ & $44.7 \pm 1.4$ & $\cdots$ & $\cdots$ & $64.2 \pm 1.3$ & $\cdots$ \\
\hline $\mathrm{C} 8$ & $36.3 \pm 1.6$ & $51.4 \pm 1.2$ & $\cdots$ & $\cdots$ & $77.1 \pm 2.6$ & $106.7 \pm 2.9$ \\
\hline C9 & $37.7 \pm 1.7$ & $48.5 \pm 9.6$ & $\cdots$ & $\cdots$ & $109.2 \pm 2.6$ & $139.6 \pm 5.7$ \\
\hline $\mathrm{C} 12$ & $54.5 \pm 3.6$ & $100.0 \pm 4.9$ & $\cdots$ & $\cdots$ & $158.6 \pm 5.7$ & $232.4 \pm 7.1$ \\
\hline $\mathrm{C14}$ & $95.4 \pm 3.5$ & $195.8 \pm 3.2$ & 101. $0 \pm 2.4$ & $185.8 \pm 4.9$ & $281.4 \pm 12.1$ & $410.1 \pm 15.8$ \\
\hline $\mathrm{C} 15$ & $138.5 \pm 3.7$ & $253.0 \pm 8.2$ & $\cdots$ & $\cdots$ & $\ldots$ & $\ldots$ \\
\hline DOPC & $23^{\circ}$ & $51^{\circ}$ & & & & \\
\hline $\mathrm{C9}$ & $13.2 \pm 1.0$ & $21.0 \pm 1.0$ & $\cdots$ & $\cdots$ & $\ldots$ & $\ldots$ \\
\hline $\mathrm{C} 10$ & $12.5 \pm 0.6$ & $20.4 \pm 0.6$ & $\ldots$ & $\cdots$ & $\ldots$ & $\ldots$ \\
\hline
\end{tabular}

\section{${ }^{\mathrm{a}} 54.4 \mathrm{MHz}$.}

${ }^{\mathrm{b}} \mathrm{G} 3$ indicates the glycerol $s n-3$ carbon; $\mathrm{C} n$ indicates a given fatty acyl chain segment.

${ }^{c}$ A verage value of three observed resonances. ${ }^{37}$

Relaxation is slightly nonexponential.

${ }^{e}$ Data refer to the 9,10 double bond of the $s n-2$ chain. 
structure and dynamics of lipid bilayers. The residual deuterium quadrupole splittings, $\Delta \nu_{Q}$, and derived order parameters, $S_{\mathrm{CD}}$, are a measure of the time averaged orientation and amplitude of the carbon-deuterium bond fluctuations, while the deuterium $T_{1}$ relaxation times and derived correlation times, $\tau_{c}$, contain the dynamic information. We emphasize that the relaxation in bilayer membranes depends on both the ordering and rate of motion. Consequently, a proper analysis of lipid bilayer dynamics using relaxation time measurements must explicitly consider the contribution of the segmental order parameter in the relaxation rate expressions.

In these respects, deuterium NMR offers several distinct advantages compared to NMR methods employing other nuclei. Since the quadrupolar interaction is the dominant relaxation mechanism, intra- and intermolecular dipolar interactions can be safely neglected. As a result, the deuterium $T_{1}$ relaxation is exclusively determined by the motion of the particular molecular segment under consideration. This enables one to derive a simple analy tical expression [Eq. (5)] for the deuterium $T_{1}$ relaxation times, in which the segment ordering and rate of reorientation are represented by independent parameters whose specific influence on the relaxation behavior can be quantitatively evaluated.

For the DPPC bilayers investigated in this study, the effect of molecular ordering on the deuterium $T_{1}$ relaxation rates is small. This is due to the fact that the order parameter $S_{C D}$ depends on the orientation of the carbon-deuterium bond vector with respect to the molecular frame, as well as the amplitude of the motional fluctuations. Thus, although parts of the bilayer are rather well ordered $\left(S_{\text {mol }} \cong 0.4\right)$, the preferential orientation of the carbon-deuterium bond vector perpendicular to the long molecular axis of the hydrocarbon chains results in a deuterium order parameter $S_{\mathrm{CD}}$ between zero and about -0.2 . Consequently, the order "correction" in Eq. (7) tends to be less than $20 \%$.

An example that is particularly well suited to demonstrate the relatively small influence of the order parameter on the $T_{1}$ relaxation rates is provided by the cisdouble bond of DOPC bilayers. As a result of the different average orientations with respect to the bilayer normal, the $\mathrm{C} 9$ and $\mathrm{C} 10$ deuterons of the cis-double bond give rise to different quadrupole splittings, corresponding to order parameters, $S_{\mathrm{CD}}$, of -0.10 and +0.02 , respectively $\left(23^{\circ} \mathrm{C}\right) .^{32}$ Since the two deuterons are attached to the same structural element, the cis-double bond, they necessarily have the same correlation time. Therefore, any differences in the relaxation times must be due to the different order parameters. Inserting the above values for $S_{C D}$ into Eq. (7) leads to a predicted ratio of $T_{1}(\mathrm{C} 9) / T_{1}(\mathrm{C} 10)=1.07$, in agreement with our experimental results, where differences in the relaxation times of the two deuterons cannot be distinguished. The short relaxation times of the 9,10 deuterons of DOPC compared to the $\mathrm{CD}_{2}$ segments of DPPC can be rationalized in terms of the larger size and correspondingly slower motion of the chain segment containing the double bond.

\section{B. Vesicles and multilayers}

Comparison of Eqs. (7) and (8) shows that for order parameters $\left|S_{C D}\right| \leq 0.2$, the maximum difference to be expected in the multilamellar and vesicle $T_{1}$ relaxation times is about $10 \%$. This result is also consistent with our experimental observations, in which significant differences in the $T_{1}$ relaxation of the vesicle and multilamellar samples are not detectable, even at those segment positions close to the glycerol backbone, which are expected to be most sensitive to the vesicle tumbling. We may therefore conclude that the fast chain segmental motions affecting $T_{1}$ are similar in the vesicle and multilamellar preparations. For both systems the macroscopic reorientation is too slow to affect the $T_{1}$ relaxation; consequently problems associated with the proper separation of local segmental motions from large-scale tumbling motions are minimal or do not exist for these systems.

\section{Bilayer profiles of the rotational correlation times and order parameters}

We now turn to a more detailed discussion of the order and rate profiles in DPPC bilayers. Since the ordering contribution is small, the plots of $1 / T_{1}$ vs the $\mathrm{CD}_{2}$ segment position largely reflect the motional profile along the hydrocarbon chains. We envisage that the molecular motions responsible for the $T_{1}$ relaxation may include chain rotational isomerizations, rotation of lipid molecules in the bilayer, and possibly any relatively low frequency modes of chain torsional oscillations. Simple torsional oscillations, stretchings, and vibrations will not contribute significantly to the relaxation, since such motions are of relatively low amplitude and occur at higher frequencies than employed in NMR experiments; likewise, any additional slow motional processes occurring at lower frequencies will be ineffective in producing $T_{1}$ relaxation. In view of the probable complexity of a detailed treatment, we have not analyzed the lipid segmental motions in terms of any discrete models, but rather have assumed that the temporal autocorrelation function decays exponentially with a single effective correlation time. As discussed elsewhere, ${ }^{48}$ the molecular fluctuations affecting the deuterium and carbon-13 $T_{1}$ relaxation in lipid bilayer systems appear to correspond to a fairly distinct class of intramolecular motions; this observation, together with the magnitude of the thermal activation barriers, suggests an interpretation of the $T_{1}$ results predominantly in terms of rotational isomerization $^{55}$ about carbon-carbon bonds. Such motions are effective in reorienting the carbon-deuterium bond vector through large solid angles and, in liquids, occur with high probability in the frequency range of NMR.

In Fig. 6 we have compared the rotational correlation times derived using Eq. (7) to the deuterium order parameters as a function of the labeled segment position. Excepting the glycerol $s n-3$ and fatty acyl C2 segments, which are discussed in the following section, the shapes of the correlation time and order profiles are similar. Both the rate of the motion, characterized by $\tau_{c}$, and the amplitude, characterized by $S_{\mathrm{CD}}$, are approximately constant from chain segments $\mathrm{C} 3$ to about $\mathrm{C} 9$. Beyond 


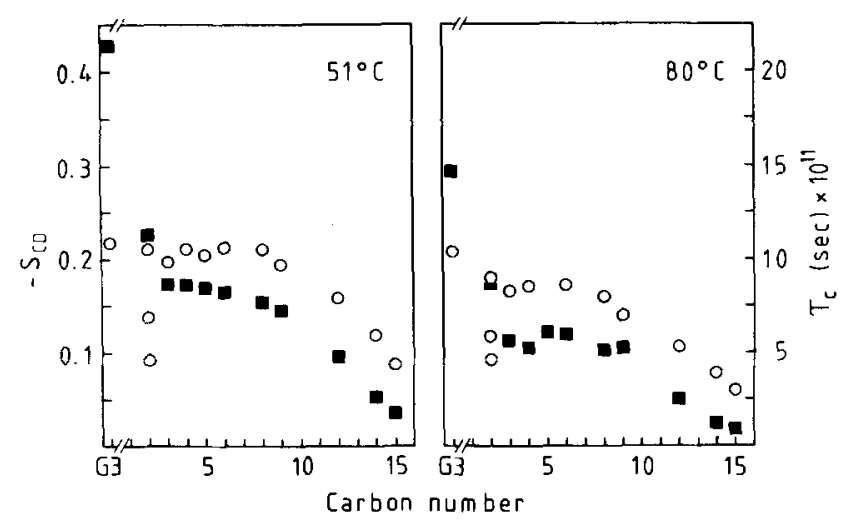

FIG, 6. Comparison of the rotational correlation times ( 1 ) determined from the $T_{1}$ data using Eq. (7) and the deuterium order parameters $(0)$ as a function of chain position. At those segment positions near the beginning or end of the fatty acyl chains, the order parameters represent the average of the two observed quadrupole splittings. ${ }^{28}$ The correlation times of the C2 segment were derived from the average relaxation rate of the three observed resonances. ${ }^{52}$

C9, a progressive increase in the rate and amplitude of the motion is evident. These features are remarkably constant over the temperature range investigated (51$80^{\circ} \mathrm{C}$ ). The fast motional fluctuations affecting $T_{1}$ occur at frequencies up to $1 / \tau_{c} \cong 10^{10} \mathrm{~Hz}$ in the first part of the fatty acyl chains, increasing to $1 / \tau_{c} \cong 10^{11} \mathrm{~Hz}$ near the terminal $\mathrm{CH}_{3}$ groups.

A quantitative analysis of the results shown in Fig. 6 will not be pursued here, but we offer the following qualitative comments. The order and correlation time profiles are best interpreted in terms of two different reference states. The order profile is most appropriately referred to the solid (gel) state with extended all-trans chains $\left(S_{\text {mol }}=1\right)$, as observed in bilayers cooled below the gel to liquid crystalline phase transition temperature. . $^{31,56}$ Above the transition temperature $\left(L_{\alpha}\right.$ phase $\left.{ }^{58}\right)$, the chain methylene segments are disordered due to trans-gauche isomerizations about various carbon-carbon bonds, but the motions are restricted in amplitude. A statistical mechanical analysis of the deuterium order parameters using the Marčelja theory ${ }^{5}$ indicates that the energetically favored conformational defects are those where a gauche rotation in one direction is accompanied by a compensating gauche rotation of the opposite sense, ${ }^{7}$ thereby preserving the parallel chain packing in the bilayer. The presence of such coupled gauche conformations (socalled kinks and jogs) provides a simple intuitive explanation of the "plateau" region of the order profile.

With regards to the relaxation rates, which measure the dynamic properties of the bilayer, the most suitable reference data are those of the liquid state. The $T_{1}$ relaxation times of DPPC dissolved in $9: 1$ chloroform/ methanol do not exhibit a plateau; instead, the relaxation rates and derived correlation times decrease more or less continuously along the fatty acyl chains (cf. Fig. 4). This behavior is characteristic of the motion of free chains attached to a center of mass undergoing overall isotropic reorientation. ${ }^{40,57}$ Since the relaxation rates are longest near the beginning of the chains, the center of mass must be displaced towards the polar head groups, which are probably anchored in a reverse micellar structure. The progressive decrease in the relaxation rates proceeding from the glycerol groups toward the methyl terminus can then be explained in terms of multiple internal rotations, e.g., trans-gauche isomerizations, which progressively accumulate in frequency along the fatty acyl chains. A similar continuous decrease is observed in the carbon-13 $T_{1}$ relaxation rates of 10-methylnonadecane, where each pair of equivalent carbon atoms in the chain is magnetically distinct and gives $r$ ise to a resolved resonance. ${ }^{58}$ Thus we can conclude that, within the confines of the rotational isomeric model, ${ }^{55}$ the motions about carbon-carbon segments in free hydrocarbon chains are essentially uncorrelated.

In the bilayer state, the fatty acyl moieties are tightly packed and prevented from behaving as isolated chains. Compared to simple paraffinic liquids, the hydrocarbon chain motion in bilayers is different in two respects. First, the chain ordering is accompanied by substantial decreases in the rate of the segmental motions compared to free hydrocarbon chains. This is evidenced by an increase in the segmental correlation times of the DPPC bilayer by a factor of up to ten compared to paraffinic liquids ${ }^{58-60}$ and by an increase in the activation energies from about $10.5 \mathrm{~kJ} \mathrm{~mol}^{-1}\left(2.5 \mathrm{kcal} \mathrm{mol}^{-1}\right)$ for DP PC in chloroform/methanol solution to about $14.6 \mathrm{~kJ} \mathrm{~mol}^{-1}$ (3. $5 \mathrm{kcal} \mathrm{mol}^{-1}$ ) in the bilayer state. Second, the observation of an approximately constant effective correlation time over almost half the length of the fatty acyl chains, i.e., a plateau is observed from chain segments $\mathrm{C} 3$ to about $\mathrm{C} 9$, suggests that the segmental motions in this region of the bilayer are rather strongly correlated. Towards the fatty acyl chain ends (C10-C15) a progressive increase in the rate of motion is observed, which presumably represents a falloff in the degree of motional correlation along the chains.

In view of the above considerations, it is clear that the effective motions of individual chain segments in lipid bilayers cannot be treated in terms of cumulative internal rotations. Rather, a more sophisticated model is required in which concerted conformational changes are explicitly taken into consideration. In the bilayer state the lipid molecules are effectively attached via their polar groups to the aqueous interface. Consequently, the van der Waals interactions among the various chain segments give rise to a lateral pressure, so that the segmental motions, while rapid, are coupled in a manner which allows for a statistical ordering of the fatty acyl chains perpendicular to the bilayer surface. From the statistical mechanical analysis of the deuterium order profiles it is found that the probabilities of the various fatty acyl chain configurations are approximately constant from near the glycerol backbone region to about the $\mathrm{C} 10$ segment, i. e., the plateau, with a decrease in the number of trans elements aligned parallel to the bilayer normal in the central hydrocarbon region. ${ }^{7}$ In view of the close connection between the correlation time and order profiles, it is possible that the constant motional component, i.e., the correlation time plateau, represents the movement of small configurational de- 
fects such as kink and jog-type conformations in the bilayer. However, a fuller understanding of the correlation time and order profiles must await the development of a more detailed model, which would presumably be the dynamic equivalent of the Marcelja theory. In our view, the experimental criteria to measure the quality of such predictive theories are now available.

\section{Segments close to the lipid-water interface}

Rather short relaxation times are observed for the deuterons of the fatty acyl C2 segments $\left(T_{1}=23 \mathrm{msec}\right.$ at $\left.51^{\circ} \mathrm{C}\right)$ and the glycerol-sn-3 segment $\left(T_{1}=13 \mathrm{msec}\right.$, $51^{\circ} \mathrm{C}$ ), compared to either the polar head group segments ${ }^{53}$ or the bulk of the hydrocarbon chain segments. Similar observations have been made previously from carbon-13 NMR studies. ${ }^{35}$ The correlation time profiles shown in Fig. 6 illustrate rather clearly that the rate of segmental motion is less at the G3 and C2 segments than deeper in the hydrocarbon region. This conclusion is further supported by the observation of a larger spectral linewidth for the G3 segment vis - $\mathrm{a}$-vis the hydrocarbon region $^{61}$ and is in agreement with previous studies indicating a relatively well defined structure for the glycerol region. ${ }^{52,62,63}$ presumably this behavior reflects the rigid anchoring and relatively tight packing of phospholipid molecules at the lipid-water boundary region, ${ }^{64}$ suggesting that the possible presence of an interfacial barrier at the level of the glycerol backbone may be a factor which should be considered with regards to the permeation of nonelectrolytes through the bilayer. The observation of similar relaxation times for the $\mathrm{C} 2$ segments of the $s n-1$ and $s n-2$ chains, which have different quadrupole splittings, ${ }^{52}$ provides further support for our contention that the rate of motion is the dominant factor in determining the relaxation of the systems investigated here.

\section{CONCLUSIONS}

The present results lead to the some rather definite ideas regarding the molecular basis of lipid bilayer membrane fluidity. The focus of previous work employing deuterium NMR has been to demonstrate the presence of well defined structural features in lipid bilayers, which appear to be general and occur in a number of membrane systems. ${ }^{63}$ The studies described here provide new information concerning the nature and rates of the intramolecular motions in lipid bilayers. For example, we can say that the configurational freedom near the glycerol backbone region is substantially reduced compared to the hydrocarbon region and that the motion of the fatty acyl chains parallels the ordering of the hydrocarbon region and thus may be related to the defect structure of the bilayer. From a dynamic point of view, the primary result of the increased orientational order in the bilyaer is not a large decrease in the rate of segmental motion compared to paraffinic liquids, but rather a correlation of the rotational motions involving neighboring segments in the fatty acyl chains. Presumably this behavior can be described in terms of a mean field bilayer pressure, which favors extension of the chains perpendicular to the bilyaer surface. The possibility of such coupled motions involving the hydrocarbon chains of lipid bilyaers has been discussed to some extent in the earlier literature $\mathrm{e}^{36,41-43}$; as directly deomonstrated here, such motional correlations appear to be an important feature of membrane organization. The rate of segmental motion in the hydrocarbon region is rapid, yet the motional correlations allow for the long range ordering characteristic of bilayer membranes.

${ }^{1}$ A. D. Bangham, Prog. Biophys. Mol. Biol. 18, 29 (1968).

${ }^{2}$ C. -h. Huang, Biochem. 8, 344 (1969).

${ }^{3}$ J. F. Nagle, J. Chem. Phys. 58, 252 (1973).

${ }^{4}$ P. Bothorel, J. Belle, and B. Lemaire, Chem. Phys. Lipids 12, 96 (1974)

${ }^{5}$ S. Marčelja, Biochim. Biophys. Acta 367, 165 (1974).

${ }^{6}$ R. E. Jacobs, B. Hudson, and H. C. Anderson, Proc. Natl. Acad. Sci. USA 72, 3993 (1975).

${ }^{7} \mathrm{H}$. Schindler and J. Seelig, Biochem. 14, 2283 (1975).

${ }^{8}$ H. L. Scott, Jr., Biochim. Biophys. Acta 469, 264 (1977).

${ }^{9}$ D. Papahadjopoulos, Biochim. Biophys. Acta 265, 169 (1972).

${ }^{10} \mathrm{~J}$. R. Trudell, Anesthesiology 46, 5 (1977).

${ }^{11}$ N. P. Franks and W. R. Lieb, Nature (London) 274, 339 (1978).

${ }^{12}$ S. G. A. McLaughlin, G. Szabo, and G. Eisenman, J. Gen. Physiol. 58, 667 (1971).

${ }^{13} \mathrm{~K}$. Jacobson and D. Papahadjopoulos, Biochem. 14, 152 (1975).

${ }^{14} \mathrm{M}$. F. Brown and J. Seelig, Nature (London) 269, 721 (1977)

${ }^{15} \mathrm{H}$. Hauser, W. Guyer, B. Levine, P. Skrabal, and R. J. P. Williams, Biochim. Biophys. Acta 508, 450 (1978).

${ }^{16}$ R. A. Haberkorn, R. G. Griffin, M. D. Meadows, and E. Oldfield, J. Am. Chem. Soc. 99, 7353 (1977).

${ }^{17} \mathrm{~S}$. Mabrey, P. L. Mateo, and J. M. Sturtevant, Biochem. 17, 2464 (1978).

${ }^{18}$ M. F. Brown and J. Seelig, Biochem. 17, 381 (1978).

${ }^{19}$ P. C. Jost, O. H. Griffith, R. A. Capaldi, and G. Vanderkooi, Proc. Natl. Acad. Sci. USA 70, 480 (1973).

${ }^{20}$ M. F. Brown, G. P. Miljanich, and E. A. Dratz, Proc. Natl. Acad. Sci. USA 74, 1978 (1977).

${ }^{21}$ M. F. Brown, G. P. Miljanich, and E. A. Dratz, Biochem. 16, $2640(1977)$

${ }^{22}$ B. A. Cornell, M. M. Sacré, W. E. Peel, and D. Chapman, FEBS Lett. 90, 29 (1978).

${ }^{23}$ E. Favre, A. Baroin, A. Bienvenue, and P. F. Devaux, Biochem. (in press)

${ }^{24} \mathrm{~A}$. Seelig and J. Seelig, Hoppe-Seyler's Z. Physiol. Chem. 359, 1747 (1978).

${ }^{25}$ H. H. Mantsch, H. Saitô, and I. C. P. Smith, Progress in NMR Spectrosc. 11, 211 (1977).

${ }^{26} \mathrm{~J}$. Seelig, Quart. Rev. Biophys. 10, 353 (1977).

${ }^{27} \mathrm{~J}$. Seelig and $\mathrm{W}$. Niederberger, Biochem. 13, 1585 (1974).

${ }^{28} \mathrm{~A}$. Seelig and J. Seelig, Biochem. 13, 4839 (1974).

${ }^{29} \mathrm{G}$. W. Stockton, C. F. Polnaszek, A. P. Tulloch, F. Hasan, and I. C. P. Smith, Biochem. 15, 954 (1976).

${ }^{30} \mathrm{~A}$. Seelig and J. Seelig, Biochem. 16, 45 (1977).

${ }^{31}$ J. H. Davis and K. R. Jeffrey, Chem. Phys. Lipids 20, 87 (1977).

${ }^{32} \mathrm{~J}$. Seelìg and N. Waespe-Šarčevié, Biochem. 17, 3310 (1978).

${ }^{33}$ E. Oldfield, M. Meadows, D. Rice, and R. Jacobs, Biochem. 17, 2727 (1978).

${ }^{34}$ A. G. Lee, N. J. M. Birdsall, Y. K. Levine, and J. C. Metcalfe, Biochim. Biophys. Acta 255, 43 (1972).

${ }^{35}$ Y. K. Levine, N. J. M. Birdsall, A. G. Lee, and J. C. Metcalfe, Biochem. 11, 1416 (1972).

${ }^{38}$ A. F. Horwitz, M. P. Klein, D. M. Michaelson, and S. J. Kohler, Ann. NY Acad. Sci. 222, 468 (1973).

${ }^{37} \mathrm{G}$. W. Feigenson and S. I. Chan, J. Am. Chem. Soc. 85, 7541 (1973).

${ }^{38}$ B. Sears, J. Membrane Biol. 20, 59 (1975). 
${ }^{39}$ A. G. Lee, N. J. M. Birdsall, J. C. Metcalfe, G. B. Warren, and G. C. K. Roberts, Proc. R. Soc. London Ser. B 193, 253 (1976).

${ }^{40} \mathrm{Y}$. K. Levine, P. Partington, and G. C. K. Roberts, Mol. Phys. 25, 497 (1973).

${ }^{41}$ M. P. N. Gent and J. H. Prestegard, J. Magn. Reson. 25, 243 (1977).

${ }^{42}$ N. O. Peterson and S. I. Chan, Biochem, 16, 2657 (1977).

${ }^{43}$ R. E. London and J. Avitable, J. Am. Chem. Soc. 99, 7765 (1977).

${ }^{44} \mathrm{~A}$. Carrington and A. D. McLachlan, Introduction to Magnetic Resonance (Harper and Row, New York, 1967), p. 191.

${ }^{45}$ S. H. Glarum and J. H. Marshall, J. Chem. Phys. 46, 55 (1967).

${ }^{46}$ M. E. Rose, Elementary Theory of Angular Momentum (Wiley, New York, 1967), Chaps. 4 and 5.

${ }^{47} \mathrm{~J}$. H. Davis, K. R. Jeffrey, and M. Bloom, J. Magn. Reson. 28, 191 (1978).

${ }^{48}$ M. F. Brown, J. Magn. Reson. (to be published).

${ }^{49}$ Abbreviations used are: DPPC, 1,2-dipalmitoyl-sn-glycero3-phosphocholine; DOPC, 1,2-dioleoyl-sn-glycero-3-phosphocholine.

${ }^{50}$ J. H. Davis, K. R. Jeffrey, M. Bloom, M. I. Valic, and T. P. Higgs, Chem. Phys, Lett. 42, 390 (1976).
${ }^{51}$ Calculated from the Bloch equations.

${ }^{52} \mathrm{~A}$. Seelig and J. Seelig, Biochim. Biophys. Acta 406, 1 (1975).

${ }^{53}$ H. U. Gally, W. Niederberger, and J. Seelig, Biochem. 14, 3647 (1975)

${ }^{54}$ J. H. Davis, Biophys. J. (in press).

${ }^{55}$ P. J. Flory, Statistical Mechanics of Chain Molecules (Wiley-Interscience, New York, 1969).

${ }^{56}$ J. L. Ranck, L. Mateu, D. M. Sadler, A. Tardieu, T. GulikKrzywicki, and V. Luzzati, J. Mol. Biol. 85, 249 (1974), and references therein.

${ }^{57}$ D. Wallach, J. Chem. Phys. 47, 5258 (1967).

${ }^{58}$ J. R. Lyerla, Jr., and T. T. Horikawa, J. Phys. Chem. 80, 1106 (1976).

${ }^{59}$ D. E. Woessner, B. S. Snowden, Jr., R. A. McKay, and E. Thomas Strom, J. Magn. Reson. 1, 105 (1969).

${ }^{60}$ Y. K. Levine, N. J. M. Birdsall, A. G. Lee, J. C. Metcalfe, P. Partington, and G. C. K. Roberts, J. Chem. Phys. 60, 2890 (1974).

${ }^{61}$ M. F. Brown, J. Seelig, and U. Haeberlen, unpublished data. ${ }^{62}$ P. B. Hitchcock, R. Mason, K. M. Thomas, and G. G. Shipley, Proc. Natl. Acad. Sci. USA 71, 3036 (1974).

${ }^{63}$ J. Seelig and J. L. Browning, FEBS Lett. 92, 41 (1978).

${ }^{64}$ G. Büldt, H. U. Gally, A. Seelig, J. Seelig, and G. Zaccai, Nature (London) 271, 182 (1978). 